\title{
So beseitigt man das Defizit
}

MMW: Zu welchen Vitamin-D-Spiegeln raten Sie Ihren Patienten?

Prof. Ringe: Als Maß für die Vitamin-D-Versorgung eines Individuums gilt der $25(\mathrm{OH}) \mathrm{D}$ Blutspiegel. Verschiedene Studien haben gezeigt, dass bei $25(\mathrm{OH}) \mathrm{D}$-Werten unter $30 \mathrm{ng} /$ $\mathrm{ml}$ bzw. $75 \mathrm{nmol} / \mathrm{l}$ der PTH-Spiegel zunehmend ansteigt. Wie auch eine Reihe anderer Experten sehe ich daher $30 \mathrm{ng} / \mathrm{ml}$ als einen vermutlich optimalen Wert an.

MMW: Wie lautet Ihre persönliche Empfehlung, um ausreichende Vitamin-D-Spiegel zu erreichen?

Prof. Ringe: Eine ausreichende Vitamin-DVersorgung ist in der deutschen Bevölkerung allein durch den Konsum Vitamin-Dreicher Nahrung und regelmäßige Sonnenexposition nicht zu schaffen. Wir konnten in einer Studie an über 1.000 Patienten nachweisen, dass am Ende der Wintermonate Alt und Jung im Mittel an einem ausgeprägten Vitamin-D-Mangel leiden. Wenn wir bei einem Patienten zu Beginn einer Beratung oder Behandlung einen sehr niedrigen Vitamin-D-Spiegel festgestellt haben, empfehlen wir in der Regel die zunächst einmalige orale Einnahme von 100.000 I.E. als "loading dose", um das weitgehend leere Depot aufzufüllen.

MMW: Reicht bei festgestelltem Vitamin-DMangel die initiale Hochdosis-Gabe von 100.000 I.E. aus, um daraufhin direkt mit einer niedrig dosierten Erhaltungstherapie (z.B. 25.000 I.E. pro Monat oder 800 I.E. pro Tag) zu beginnen?

Prof. Ringe: Dies hängt vom Einzelfall ab. Wenn ein Patient seinen Lebensstil nach erster Feststellung eines starken Vitamin-DMangels ändert - z. B. mehr Vitamin D mit der Ernährung, mehr Sonnenexposition -

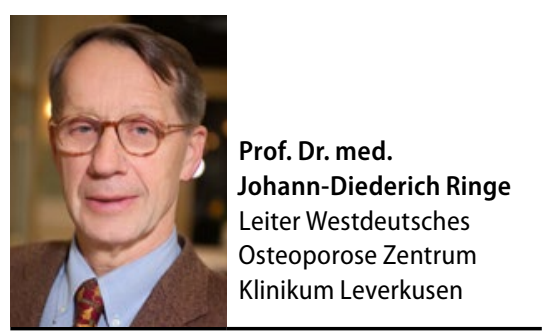

könnte eine einmalige Hochdosis ausreichen. Im Zweifelsfall müsste jedoch der 25(OH)D-Spiegel kontrolliert werden, um zu prüfen, ob z.B. nach sechs Monaten die tägliche oder monatliche Erhaltungsdosis ausreicht oder der Vitamin-D-Spiegel wieder absinkt. Dann wäre ggf. die Initialdosis zu wiederholen und Erhaltungsdosis höher einzustellen.

Red.

- Nach Informationen von InfectoPharm Arzneimittel und Consilium $\mathrm{GmbH}$

\section{Antikoagulation bei Beinvenenthrombose}

\section{Ein halbes Jahr ist oft nicht genug}

_ Die venöse Thromboembolie (VTE) durchläuft drei Phasen, die akute in den ersten vier Wochen, die mittelfristige über drei bis sechs Monate und die langfristige nach sechs Monaten. Die bisherige Standardtherapie war die Gabe eines niedermolekularen Heparins über mindestens fünf Tage, gefolgt von einer Erhaltungstherapie mit einem VitaminK-Antagonisten über drei bis sechs Monate. „Das Rezidivrisiko ist jedoch im ersten Jahr nach Beendigung der Antikoagulation am höchsten", erläuterte Prof. Edelgard Lindhoff-Last, Frankfurt am Main.

Die primäre symptomatische Lungenembolie und die proximalen Thrombosen haben das höchste Rezidivrisiko, so Lindhoff-Last. Bei Patienten mit einem erhöhten Rezidivrisiko empfehle sich deshalb eine verlängerte Sekundärprophylaxe. Der Nutzen einer solchen wurde in der AMPLIFY-EXT-Studie gegenüber Placebo belegt. Die Patienten erhielten randomisiert nach der initialen 6- bis 12-monatigen Antikoagulation entweder Apixaban oder Placebo über weitere 12 Monate. Mit Apixaban wurde der primäre Wirksamkeitsendpunkt (symptomatische, rezidivierende VTE oder Tod jeglicher Ursache) von 9,3\% unter Placebo auf 2,3\% gesenkt. Die gute Wirksamkeit ging auch in dieser Studie mit einem überzeugenden Sicherheitsprofil einher, d. h. die Rate schwerer Blutungen lag im Placebobereich.

\section{Dr. Peter Stiefelhagen}

- Symposium „Lungenembolie und tiefe BeinvenenthromboseDiagnostische und therapeutische Tipps für den internistischen Alltag", 123. Kongress der Deutschen Gesellschaft für Innere Medizin; Mannheim, April 2017 (Veranstalter: BMS und Pfizer)

\section{Interleukin-Inhibitor hilft beim periodischen Fiebersyndrom}

Zu den autoinflammatorischen Erkrankungen gehören die periodischen Fiebersyndrome (PFS). Sie sind charakterisiert durch wiederkehrende Fieberschübe mit potenziell lebensbedrohlichen Komplikationen. Ursache der PFS, zu denen auch das familiäre Mittelmeerfieber (FMF) zählt, sind Genvarianten, die zu einer übermäßigen Aktivierung des angeborenen Immunsystems mit pathologisch gesteigerter Entzündungsreaktion führen. Dies führt zu einer Überproduktion von Interleukin-1 $\beta$ (IL-1ß). Laut EULAR-Empfehlungen ist eine altersabhängig dosierte Colchizin-Therapie das Vorgehen der Wahl bei FMF. Bei Colchizin-Resistenz oder -Unverträglichkeit (jeweils 5-10\% der Fälle) können Interleukin-Inhibitoren wie Canakinumab eingesetzt werden.

Christina Ott

- Satellitensymposium „Autoinflammation im Kindes- und Erwachsenenalter - Neues und Bewährtes", 31. Kongress der Deutschen Gesellschaft für Rheumatologie; Stuttgart, September 2017 (Veranstalter: Novartis) 